\title{
Brownian motion in a granular gas
}

\author{
J. Javier Brey, M. J. Ruiz-Montero, and R. García-Rojo \\ Física Teórica, Universidad de Sevilla, E-41080 Sevilla, Spain \\ James W. Dufty \\ Department of Physics, University of Florida, Gainesville, Florida 32611
}

(Received 2 June 1999)

\begin{abstract}
The dynamics of a heavy particle in a gas of much lighter particles is studied via the Boltzmann-Lorentz equation with inelastic collisions among all particles. A formal expansion in the ratio of gas to tagged particle mass transforms the Boltzmann-Lorentz equation into a Fokker Planck equation. The predictions of the latter are tested here using direct Monte Carlo simulation of the Boltzmann-Lorentz equation. Excellent agreement is obtained for the approach to a homogeneous cooling state, the temperature of that state, approach to diffusion, and the dependence of the diffusion constant on dissipation parameters. Some results from molecular-dynamics simulations are also presented and shown to agree with the theoretical predictions. [S1063-651X(99)08012-5]
\end{abstract}

PACS number(s): 81.05.Rm, 05.20.Dd, 05.40.-a

\section{INTRODUCTION}

The description of low-density, rapid granular flow by Boltzmann kinetic theory has received much attention in recent years $[1,2]$. A primary objective has been the derivation of hydrodynamic equations and expressions for the transport coefficients appearing in them as functions of the coefficient of restitution. The most accurate approach uses a generalization of the Chapman-Enskog method known from the corresponding analysis of the Boltzmann equation for elastic collisions $[3,4]$. This method assumes the existence of a "normal" solution whose space and time dependence occurs only through the hydrodynamic fields. Implicit in this assumption is the rapid relaxation of nonhydrodynamic excitations so that the hydrodynamic description dominates on a longer time scale. Such a separation of time scales has been questioned in the case of granular flow, particularly for large degrees of inelasticity [5]. The basis for this concern seems to be the additional time scale set by the cooling of the homogeneous reference state, such that the hydrodynamic time scales are not simply determined by the degree of spatial inhomogeneity. However, detailed study of self-diffusion [6] and kinetic models based on the Boltzmann equation [7] suggests this concern is not justified. For these cases it is shown that the microscopic excitations always decay on times short compared to all hydrodynamic times, including that defined by the cooling rate.

To further reinforce the case for a hydrodynamic description, an exact analysis of the Boltzmann-Lorentz kinetic equation for a tagged particle in a freely evolving gas has been performed in the limit of asymptotically large relative mass for the tagged particle [7]. In this limit, the BoltzmannLorentz equation reduces to a Fokker-Planck equation. An exact analysis of the spectrum for this equation confirms the separation of time scales for all degrees of tagged particle inelasticity. It remains to confirm the validity of the formal asymptotic analysis leading from the Boltzmann-Lorentz equation to the Fokker-Planck equation. One objective here is to provide this confirmation on the basis of direct Monte Carlo simulation of the Boltzmann-Lorentz equation.
In the next section the Boltzmann-Lorentz and its FokkerPlanck limit are recalled. A change of space and time variables provides an exact map of the Fokker-Planck equation for inelastic collisions to that for elastic collisions. Consequently, all the known results from the latter case for velocity relaxation and the approach to a diffusive stage translate exactly to the case of inelastic collisions. Several examples are considered explicitly. The qualitative differences occur only through the changes in space and time scales. Some of the most interesting differences are as follows.

(i) The renormalized time scale is related logarithmically to real time. Accordingly, velocity relaxation and approach to hydrodynamics is algebraic rather than exponential.

(ii) The long time limit of the tagged particle distribution for the homogeneous state is Gaussian, although the bath particle distribution is non-Gaussian.

(iii) The time-dependent temperature of the tagged particle Gaussian differs from the temperature of the surrounding bath, although the cooling rates of both become the same at long times.

(iv) The mean-square displacement approaches $\ln t$ for large times with a coefficient that determines the diffusion coefficient.

(v) The diffusion equation at long times has the usual form, although its solution is qualitatively different from that for the elastic case due to the time dependence of the temperature in the diffusion coefficient.

In Sec. III the direct simulation Monte Carlo method to obtain numerical solutions of the Boltzmann equation is described briefly. Comparisons are made between the simulations of the Boltzmann-Lorentz equation and the predictions of the Fokker-Planck equation for a tagged particle whose mass is 100 times that of the surrounding gas particles. The Maxwellian distribution is confirmed, as is the approach to different temperatures for the bath and tagged particle at the same cooling rates. The mean-square displacement is measured and the diffusion coefficient extracted from its long time behavior to confirm the detailed dependence on inelasticity as predicted by the Fokker-Planck equation. The results provide strong support for the accuracy of the Fokker-Planck 
equation as a representation of the Boltzmann-Lorentz equation for large relative mass.

Of course, all the above discussion relies on the validity of the (inelastic) Boltzmann-Lorentz equation to describe a massive tagged particle in a freely evolving gas. While this validity is well established for elastic collisions, it is sometimes questioned for inelastic collisions. To provide support for this case, we have performed molecular-dynamics simulations for this system in two dimensions. The results presented in Sec. IV confirm the analysis based on the kinetic equation, indicating clearly the validity of kinetic equations to study rapid granular flows. This section also includes a summary and discussion of the main results in the paper.

\section{FOKKER-PLANCK EQUATION AND ITS SOLUTION}

A tagged particle immersed in a low-density gas is considered. The gas is formed by hard spheres $(d=3)$ or disks $(d=2)$ of mass $m_{g}$ and diameter $\sigma_{g}$, and the tagged particle is also a hard sphere or disk, but with mass $m$ and diameter $\sigma$. All particles are smooth and collide inelastically. Collisions are characterized by velocity-independent coefficients of normal restitution. For the collisions between the gas particles it will be denoted by $\alpha_{g}$, while that for collisions between the tagged particle and gas particles will be represented by $\alpha$.

The probability density $F(\mathbf{r}, \mathbf{v}, t)$ describing the dynamics of the tagged particle obeys the Boltzmann-Lorentz equation

$$
\left(\partial_{t}+\mathbf{v} \cdot \boldsymbol{\nabla}\right) F=J[\mathbf{r}, \mathbf{v}, t \mid F, f] .
$$

The collision operator $J$ is given by $[7,8]$

$$
\begin{aligned}
J[\mathbf{r}, \mathbf{v}, t \mid F, f]= & \sigma_{0}^{d-1} \int d \mathbf{v}_{1} \int d \hat{\boldsymbol{\sigma}} \Theta(\mathbf{g} \cdot \hat{\boldsymbol{\sigma}})(\mathbf{g} \cdot \hat{\boldsymbol{\sigma}}) \\
& \times\left[\alpha^{-2} F\left(\mathbf{r}, \mathbf{v}^{\prime}, t\right) f\left(\mathbf{r}, \mathbf{v}_{1}^{\prime}, t\right)\right. \\
& \left.-F(\mathbf{r}, \mathbf{v}, t) f\left(\mathbf{r}, \mathbf{v}_{1}, t\right)\right]
\end{aligned}
$$

where $f(\mathbf{r}, \mathbf{v}, t)$ is the corresponding distribution for the surrounding gas particles, $\Theta$ is the Heaviside step function, $\hat{\boldsymbol{\sigma}}$ is a unit vector pointing from the center of the gas particle 1 to the center of the tagged particle at contact, and $\sigma_{0}=(\sigma$ $\left.+\sigma_{g}\right) / 2$. The precollisional or restituting velocities $\mathbf{v}^{\prime}$ and $\mathbf{v}_{1}^{\prime}$ are given by

$$
\mathbf{v}^{\prime}=\mathbf{v}-\frac{(1+\alpha) \Delta}{\alpha(1+\Delta)}(\mathbf{g} \cdot \hat{\boldsymbol{\sigma}}) \hat{\boldsymbol{\sigma}}, \quad \mathbf{v}_{1}^{\prime}=\mathbf{v}_{1}+\frac{1+\alpha}{\alpha(1+\Delta)}(\mathbf{g} \cdot \hat{\boldsymbol{\sigma}}) \hat{\boldsymbol{\sigma}},
$$

with $\mathbf{g}=\mathbf{v}-\mathbf{v}_{1}$ the relative velocity and $\Delta=m_{g} / m$ the ratio of gas to tagged particle mass. The surrounding gas is taken to be in its homogeneous cooling state (HCS) as determined from the solution to the nonlinear Boltzmann equation. Its distribution function has the scaling form [1]

$$
f_{H}(\mathbf{v}, t)=n_{g} \mathbf{v}_{g}^{-d}(t) \phi\left(\frac{\mathrm{v}}{\mathbf{v}_{g}(t)}\right),
$$

where $n_{g}$ is the constant number density of the gas, $\mathrm{v}_{g}(t)$ $=\left[2 k_{B} T_{g}(t) / m_{g}\right]^{1 / 2}$ is the thermal velocity of the gas par- ticles at time $t$ with $k_{B}$ being the Boltzmann constant, and $T_{g}(t)$ is the temperature of the gas which cools according to the equation

$$
\frac{d T_{g}(t)}{d t}=-\zeta(t) T_{g}(t)
$$

The cooling rate $\zeta(t)$ depends on time only through $T_{g}(t)$ and is determined from the second moment of the Boltzmann equation for the gas [8],

$$
\begin{aligned}
\zeta(t)= & \left(1-\alpha_{g}^{2}\right) \frac{n_{g} \sigma_{g}^{d-1} \mathbf{v}_{g}(t) \pi^{(d-1) / 2}}{2 d \Gamma\left(\frac{d+3}{2}\right)} \\
& \times \int d \mathbf{v} \int d \mathbf{v}_{1} g^{3} \phi(\mathbf{v}) \phi\left(\mathbf{v}_{1}\right) .
\end{aligned}
$$

The solution to Eq. (2.5) is

$$
T_{g}(t)=T_{g}(0)\left[1+\frac{\zeta(0)}{2} t\right]^{-2}
$$

showing that the temperature of the gas decreases as $t^{-2}$ for large times if the system remains in the HCS. The explicit form of $\phi$ will be not given here, but it is known in the so-called first Sonine approximation $[1,9]$.

In Ref. [7] it was shown that the Boltzmann-Lorentz equation reduces to a Fokker-Planck equation for asymptotically small $\Delta$,

$$
\left(\partial_{t}+\mathbf{v} \cdot \boldsymbol{\nabla}\right) F(\mathbf{r}, \mathbf{v}, t)=\gamma_{e}(t) a \frac{\partial}{\partial \mathbf{v}} \cdot\left[\mathbf{v}+\frac{k_{B} T_{g}(t)}{m} a \frac{\partial}{\partial \mathbf{v}}\right] F(\mathbf{r}, \mathbf{v}, t),
$$

where $\gamma_{e}(t)$ is the same friction coefficient as for elastic collisions, except as a function of the time-dependent temperature $T_{g}(t)$,

$$
\gamma_{e}(t)=\frac{4 \pi^{(d-1) / 2} \sigma_{0}^{d-1} \Delta}{d \Gamma(d / 2)} n_{g} \vee_{g}(t)
$$

where $\mathrm{v}_{g}(t)$ is defined in terms of $T_{g}(t)$ following Eq. (2.4) above. All effects of inelastic collisions among the gas particles in Eq. (2.8) appear through the time dependence of $T_{g}(t)$, while the inelasticity of collisions between the tagged particle and gas particles manifests itself only through the parameter $a=(1+\alpha) / 2$.

The Fokker-Planck equation (2.8) can be mapped onto the corresponding equation for elastic collisions using the dimensionless variables

$$
\begin{gathered}
\mathbf{v}^{*}=\frac{\mathbf{v}}{\mathbf{v}_{0}(t)}, \quad \mathbf{r}^{*}=a(1-\epsilon) \frac{\gamma_{e}(t)}{\mathbf{v}_{0}(t)} \mathbf{r} \\
t^{*}=a(1-\epsilon) \int_{0}^{t} d t^{\prime} \gamma_{e}\left(t^{\prime}\right)
\end{gathered}
$$

where we have introduced 


$$
\mathrm{v}_{0}(t)=\left(\frac{a}{1-\epsilon}\right)^{1 / 2}\left[\frac{2 k_{B} T_{g}(t)}{m}\right]^{1 / 2}, \quad \epsilon=\frac{\zeta(t)}{2 a \gamma_{e}(t)}
$$

Since both $\zeta(t)$ and $\gamma_{e}(t)$ are proportional to $T_{g}^{1 / 2}(t)$, it follows that $\epsilon$ is a time-independent quantity. The above definitions apply only for $\epsilon<1$, a point discussed further in the final section. Here we only note that it is a necessary condition in the derivation of Eq. (2.8). In terms of the new variables, the Fokker Planck equation (2.8) becomes

$$
\begin{aligned}
\left(\partial_{t^{*}}+\mathbf{v}^{*} \cdot \frac{\partial}{\partial \mathbf{r}^{*}}\right) F^{*}\left(\mathbf{r}^{*}, \mathbf{v}^{*}, t^{*}\right) \\
=\frac{\partial}{\partial \mathbf{v}^{*}} \cdot\left(\mathbf{v}^{*}+\frac{1}{2} \frac{\partial}{\partial \mathbf{v}^{*}}\right) F^{*}\left(\mathbf{r}^{*}, \mathbf{v}^{*}, t^{*}\right),
\end{aligned}
$$

with the scaled probability density $F^{*}$ given by

$$
F^{*}\left(\mathbf{r}^{*}, \mathbf{v}^{*}, t^{*}\right)=\frac{\mathbf{v}_{0}^{2 d}(t)}{\left[a(1-\epsilon) \gamma_{e}(t)\right]^{d}} F(\mathbf{r}, \mathbf{v}, t),
$$

which is also normalized to unity. Equation (2.12) is the same as the dimensionless form of the Fokker-Planck equation for elastic collisions $\left(\alpha=\alpha_{g}=1\right)$. Consequently, the physical properties of a massive tagged particle with inelastic collisions moving in a gas in the HCS are the same as those for an elastic particle in an equilibrium gas; the only differences are the relevant space and time scales. For example, $t^{*}=\gamma_{e} t$ for elastic collisions while for inelastic collisions the relationship is

$$
t^{*}=\frac{1-\epsilon}{\epsilon} \ln \left[1+\frac{\zeta(0)}{2} t\right]
$$

and the time scale is stretched logarithmically.

The general solution of Eq. (2.12) to the initial value problem for an unbounded system is well known [10]:

$$
\begin{aligned}
F^{*}\left(\mathbf{r}^{*}, \mathbf{v}^{*}, t^{*}\right)= & \int d \mathbf{r}^{*^{\prime}} \int d \mathbf{v}^{*^{\prime}} G^{*}\left(\mathbf{r}^{*}, \mathbf{v}^{*}, t^{*} ; \mathbf{r}^{*^{\prime}}, \mathbf{v}^{*^{\prime}}, 0\right) \\
& \times F^{*}\left(\mathbf{r}^{*^{\prime}}, \mathbf{v}^{*^{\prime}}, 0\right),
\end{aligned}
$$

with

$$
\begin{aligned}
G^{*}\left(\mathbf{r}^{*}, \mathbf{v}^{*}, t^{*} ; \mathbf{r}^{*^{\prime}}, \mathbf{v}^{*^{\prime}}, 0\right) \\
=\left[\frac{e\left(t^{*}\right) b\left(t^{*}\right)}{\pi^{2}}\right]^{d / 2} \exp \left\{-e\left(t^{*}\right)\left[\mathbf{r}^{*}-\mathbf{r}^{*^{\prime}}-c\left(t^{*}\right)\right.\right. \\
\left.\left.\quad \times\left(\mathbf{v}^{*}+\mathbf{v}^{* \prime}\right)\right]^{2}-b\left(t^{*}\right)\left(\mathbf{v}^{*}-e^{-t^{*}} \mathbf{v}^{*^{\prime}}\right)^{2}\right\} .
\end{aligned}
$$

In the above expressions we have defined

$$
\begin{gathered}
e\left(t^{*}\right)=\frac{1}{2\left[t^{*}-2 c\left(t^{*}\right)\right]}, \quad b\left(t^{*}\right)=\frac{1}{1-e^{-2 t^{*}},} \\
c\left(t^{*}\right)=\frac{1-e^{-t^{*}}}{1+e^{-t^{*}}} .
\end{gathered}
$$

Consider first a spatially homogeneous initial state for the tagged particle $F^{*}\left(\mathbf{r}^{*}, \mathbf{v}^{* \prime}, 0\right)=F^{*}\left(\mathbf{v}^{* \prime}, 0\right)$. Then Eq. (2.15) simplifies to

$$
\begin{aligned}
F^{*}\left(\mathbf{v}^{*}, t^{*}\right)= & {\left[\frac{b\left(t^{*}\right)}{\pi}\right]^{d / 2} \int d \mathbf{v}^{*^{\prime}} \exp \left[-b\left(t^{*}\right)\right.} \\
& \left.\times\left(\mathbf{v}^{*}-e^{-t^{*}} \mathbf{v}^{*^{\prime}}\right)^{2}\right] F^{*}\left(\mathbf{v}^{*^{\prime}}, 0\right) .
\end{aligned}
$$

For $t^{*} \gg 1, b\left(t^{*}\right) \rightarrow 1$ and, consequently,

$$
F^{*}\left(\mathbf{v}^{*}, t^{*}\right) \rightarrow F_{M}^{*}\left(\mathrm{v}^{*}\right)=\frac{1}{\Omega^{*}} \pi^{-d / 2} e^{-\mathrm{v}^{* 2}},
$$

where $\Omega^{*}$ is the volume of the system measured in the reduced length scale defined in Eq. (2.10). Thus, for general homogeneous initial conditions, the distribution function approaches exponentially fast a stationary Maxwellian distribution in the reduced units. In terms of the original variables this result is

$$
F(\mathbf{v}, t) \rightarrow F_{M}(\mathrm{v}, t)=\frac{1}{\Omega}\left[\frac{m}{2 \pi k_{B} T_{\infty}(t)}\right]^{d / 2} e^{-m v^{2} / 2 k_{B} T_{\infty}(t)},
$$

with the temperature parameter $T_{\infty}(t)$ given by

$$
T_{\infty}(t)=T_{g}(t) \frac{a}{1-\epsilon}
$$

Interestingly, the tagged particle approaches a homogeneous cooling state with a scaling form similar to Eq. (2.4) for the gas, but with two important differences. First, the cooling state for the tagged particle is Gaussian while that for the gas is not. Second, the cooling temperature for the tagged particle is different from that for the gas, although the cooling rates are the same. To elaborate on this latter feature, it is convenient to define more generally the kinetic temperature for the tagged particle by

$$
\begin{aligned}
\frac{d}{2} k_{B} T(t) & =\int d \mathbf{r} \int d \mathbf{v} \frac{1}{2} m \mathbf{v}^{2} F(\mathbf{r}, \mathbf{v}, t) \\
& =\frac{k_{B} T_{g}(t) a}{1-\epsilon} \int d \mathbf{r}^{*} \int d \mathbf{v}^{*} \mathrm{v}^{* 2} F^{*}\left(\mathbf{r}^{*}, \mathbf{v}^{*}, t\right) .
\end{aligned}
$$

Evaluating the integral using Eq. (2.15) gives

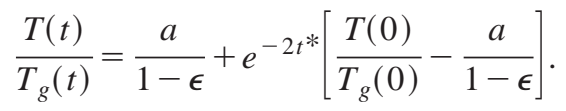

The approach of the tagged particle temperature to the asymptotic value $T_{\infty}(t)$ is exponentially fast on the reduced time scale. Note that $T_{\infty}(t) \neq T_{g}(t)$ even in the case of elastic collisions for the tagged particle $(\alpha=1)$, if the gas particle collisions are inelastic. Conversely, if gas particle collisions are elastic but the tagged particle collisions are inelastic, then $\epsilon=0$ and the tagged particle approaches the constant temperature of the gas, but still differs from it by a factor of $a$. 
The result in Eq. (2.23) applies even for arbitrary inhomogeneous states of the Brownian particle.

Consider next an initial state whose velocity distribution is given by the homogeneous cooling Maxwellian but with a spatial inhomogeneity,

$$
F^{*}\left(\mathbf{r}^{*}, \mathbf{v}^{*}, 0\right)=n^{*}\left(\mathbf{r}^{*}, 0\right) \pi^{-d / 2} e^{-\mathbf{v}^{* 2}},
$$

where $n^{*}\left(\mathbf{r}^{*}, 0\right)$ is the probability density for finding the tagged particle at position $\mathbf{r}^{*}$ at time $t=0$. The corresponding quantity at time $t^{*}$ is obtained by integration of $F^{*}\left(\mathbf{r}^{*}, \mathbf{v}^{*}, t^{*}\right)$, as given in Eq. (2.15), with respect to $\mathbf{v}^{*}$, giving

$$
\begin{aligned}
n^{*}\left(\mathbf{r}^{*}, t^{*}\right)= & {\left[\frac{d}{2 \pi l^{* 2}\left(t^{*}\right)}\right]^{d / 2} \int d \mathbf{r}^{*^{\prime}} } \\
& \times \exp \left[-d\left(\mathbf{r}^{*}-\mathbf{r}^{*^{\prime}}\right)^{2} / 2 l^{* 2}\left(t^{*}\right)\right] n^{*}\left(\mathbf{r}^{*^{\prime}}, 0\right),
\end{aligned}
$$

where

$$
l^{* 2}\left(t^{*}\right)=d\left(t^{*}-1+e^{-t^{*}}\right) .
$$

It is easily verified that $l^{* 2}\left(t^{*}\right)$ is the mean-square displacement of the tagged particle in terms of the dimensionless variables. Moreover, it follows directly from Eq. (2.25) that $n^{*}\left(\mathbf{r}^{*}, t^{*}\right)$ obeys the extended diffusion equation

$$
\partial_{t *} n^{*}\left(\mathbf{r}^{*}, t^{*}\right)=\left(1-e^{-t^{*}}\right) D^{*} \boldsymbol{\nabla}^{* 2} n^{*}\left(\mathbf{r}^{*}, t^{*}\right),
$$

with $D^{*}=1 / 2$. This equation is exact for all times if the initial condition has the assumed form, and shows most directly the approach to a hydrodynamic stage, i.e., the usual diffusion equation applies exponentially fast for $t^{*} \gg 1$. The value $D^{*}=1 / 2$ is consistent with the Einstein result

$$
D^{*}=\frac{1}{2 d} \lim _{t^{* \rightarrow \infty}} t^{*-1} l^{* 2}\left(t^{*}\right)
$$

In terms of the original variables, the asymptotic diffusion equation is

$$
\partial_{t} n(\mathbf{r}, t)=D(t) \nabla^{2} n(\mathbf{r}, t),
$$

with

$$
D(t)=\frac{D_{e}(t)}{(1-\epsilon)^{2}} .
$$

Here $D_{e}(t)=k_{B} T_{g}(t) / m \gamma_{e}(t)$ is the same as the diffusion coefficient for elastic collisions, except as a function of the time-dependent gas temperature. For a given temperature, the diffusion coefficient is seen to be enhanced by a factor of $(1-\epsilon)^{-2}$, depending only on the inelasticity of gas collisions.

For more general initial conditions, the dynamics is qualitatively similar with rapid velocity relaxation followed by spatial diffusion. In the next section, several predictions from this Fokker-Planck analysis are tested against direct simulation of the corresponding property from the BoltzmannLorentz equation.

\section{MONTE CARLO SIMULATION OF THE BOLTZMANN-LORENTZ EQUATION}

To test the formal analysis leading from the BoltzmannLorentz equation to the Fokker-Planck, the direct simulation Monte Carlo (DSMC) method [11] has been applied to the former. Specifically, the predictions of velocity relaxation to a Maxwellian at a new temperature and the approach to diffusion with an enhanced diffusion coefficient have been investigated for a system of hard spheres with mass ratio $\Delta$ $=10^{-2}$, equal diameters for all particles, and a coefficient of normal restitution for collisions between gas particles $\alpha_{g}$ $=0.99$. These values assure the necessary condition $\epsilon<1$ for $0<\alpha \leqslant 1$, as shown in the Appendix. The DSMC for the Boltzmann equation with elastic collisions is well described in Ref. [11]. Its adaptation to the Boltzmann-Lorentz equation with inelastic collisions is straightforward. As indicated in Eq. (2.1), the distribution function for the gas, $f_{s}$, is required for input, and is taken here to be the homogeneous cooling solution to the Boltzmann equation $f_{H}$. It is known that this solution is unstable to long-wavelength spatial perturbations at sufficiently long times, so any possible interference of relaxation processes by this instability is not addressed. However, for the chosen value of $\alpha_{g}$ the homogeneous cooling state is stable on the time scales studied [12].

In the simulations $10^{5}$ trajectories of a tagged particle have been generated. Independently of its position, collisions of the tagged particle always took place with particles of a homogeneous gas whose velocity distribution was given by Eq. (2.4). As already mentioned, the exact form of $\phi$ is not known, and the expression obtained in the first Sonine approximation [1,9] was used to generate the velocity of the colliding gas particles. Thus, the trajectories of the gas particles are not required, which increases greatly the efficiency of the numerical simulation and avoids the introduction of specific boundary conditions - the system is formally considered as infinite. A similar method already has been used to study self-difussion in a low-density granular flow [6].

In a typical run, collisions between the tagged particle and the fluid particles were considered as uncoupled during a time step $t_{0}$, chosen much smaller than the initial average collision time of the tagged particle. This means that the position of the tagged particle was changed at constant velocity for an interval $t_{0}$ between every two applications of the collision algorithm. For the collisions, the velocities of the particles were generated from the HCS distribution, as mentioned above, with a gas temperature determined from the law given by Eq. (2.5) and considered to be constant in the time interval $t_{0}$. This implies that $t_{0}$ has to be chosen also much smaller than the time characterizing the cooling of the gas, $2 / \zeta(0)$. In the simulations we report here we used $t_{0}$ $\simeq 2 \times 10^{-4} / \zeta(0)$. The initial condition in all simulations was a Gaussian velocity distribution with the same temperature as the surrounding fluid.

As a first test of the Fokker-Planck limit, the predicted approach to a Gaussian cooling state is studied. The associ- 


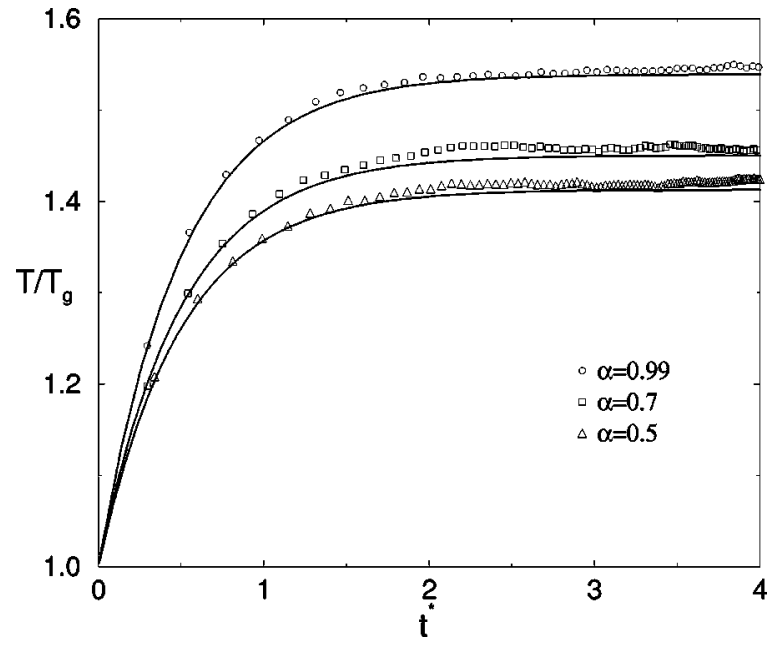

FIG. 1. Plot of the ratio of the tagged particle temperature to the surrounding gas temperature as a function of the dimensionless reduced time $t^{*}$ defined in Eq. (2.10). The ratio of gas to tagged particle mass is $10^{-2}$ and the coefficient of restitution for the gas collisions is $\alpha_{g}=0.99$. The solid lines are the predictions from the Fokker-Planck equation and the symbols from the DSMC method of the Boltzmann-Lorentz equation.

ated scaling velocity is defined in terms of the temperature $T(t)$ of the tagged particle, characterizing its kinetic energy. It is expected that this temperature approaches an asymptotic value proportional to the gas temperature $T_{g}(t)$, according to Eq. (2.23). Figure 1 shows the time evolution of the ratio $T(t) / T_{g}(t)$ as obtained from the numerical simulation of the Boltzmann-Lorentz equation for $\alpha=0.5,0.7$, and 0.99. In all cases it is seen that the ratio evolves from the unit initial condition until reaching a steady value. Also plotted is the theoretical prediction. Figure 2 shows a detailed comparison of the numerical asymptotic values with Eq. (2.21) over this range of values of $\alpha$. The agreement is very good considering that corrections to the Fokker Planck limit are of order

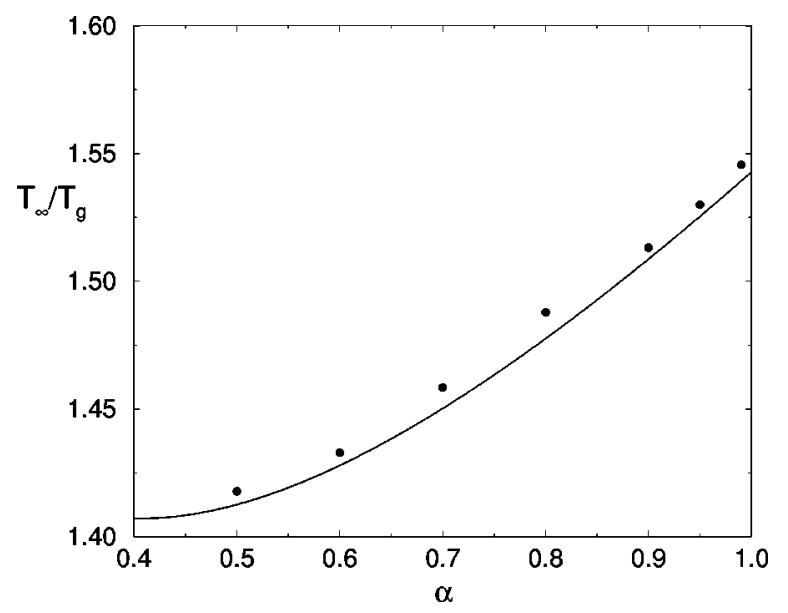

FIG. 2. Asymptotic value of the ratio between the tagged particle temperature $T$ and the gas temperature $T_{g}$ as a function of the coefficient of normal restitution $\alpha$ for collisions between the tagged particle and the fluid particles. The mass ratio and the coefficient of restitution for gas collisions are the same as in Fig. 1. The solid line is from the Fokker-Planck equation and the dots from the numerical simulation of the Boltzmann-Lorentz equation.

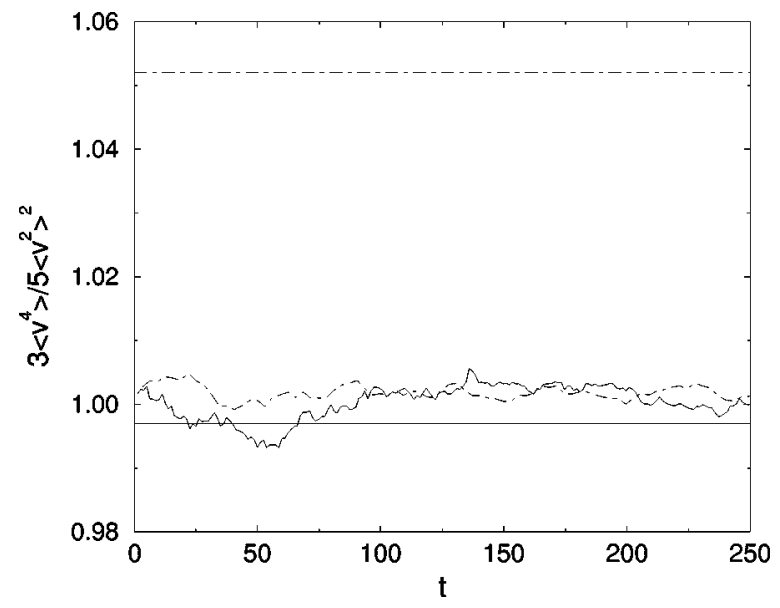

FIG. 3. Time evolution of the reduced fourth velocity moment of the tagged particle distribution. Here time is measured in units of $\lambda / \mathrm{v}_{0}$, where $\lambda$ is the mean free path. The continuous line corresponds to $\alpha=0.95$ and the dashed line to $\alpha=0.5$. The straight lines represent the fourth moment of the HCS distribution for the fluid at $\alpha=0.95$ (continuous) and $\alpha=0.5$ (dashed).

$\Delta^{1 / 2}$. Interestingly, we have found that the agreement between theory and simulations improves significantly if the mass ratio $\Delta$ and $\alpha_{g}$ are changed to reduce the value of $\epsilon$.

The Gaussian character of the tagged particle distribution can be studied via its fourth moment, or the normalized expression $3\left\langle\mathrm{v}^{4}\right\rangle / 5\left\langle\mathrm{v}^{2}\right\rangle^{2}$, which has the value unity for a Gaussian. Here it is

$$
\left\langle\mathbf{v}^{n}\right\rangle=\frac{1}{\Omega} \int d \mathbf{r} \int d \mathbf{v} \mathbf{v}^{n} F(\mathbf{r}, \mathbf{v}, t)
$$

Figure 3 shows the simulation values for the normalized moment as a function of time for $\alpha=0.5$ and 0.99. Also shown are the corresponding results for the gas distribution, i.e., for the velocity distribution in Eq. (2.4). The latter shows significant deviations from unity whereas the tagged particle results confirm the Gaussian even at strong dissipation. Although we have plotted in the figure the results corresponding to two extreme values of $\alpha$, a similar behavior has been obtained also for several intermediate values. It could be claimed that the Gaussian distribution observed in the simulations is in some way influenced by the initial Gaussian distribution. To clarify this point we have also considered initial distributions far from the Gaussian, namely a uniform distribution with zero mean. It was observed that the distribution evolved towards a Gaussian very fast, indicating that the use of an initial Gaussian does not limit at all the results presented here.

The approach to diffusion can be studied via the meansquare displacement of the tagged particle. In Fig. 4, a comparison of the simulations with the universal form given by Eq. (2.26) for $\alpha=0.5,0.7$, and 0.99 is presented. The results confirm both the approach to diffusion and the predicted limiting form with $D^{*}=1 / 2$. Figure 5 shows a more detailed comparison with the predicted diffusion coefficient over a wide range of $\alpha$ values. Again, the agreement is very good, since the discrepancies are smaller than $2 \%$. 


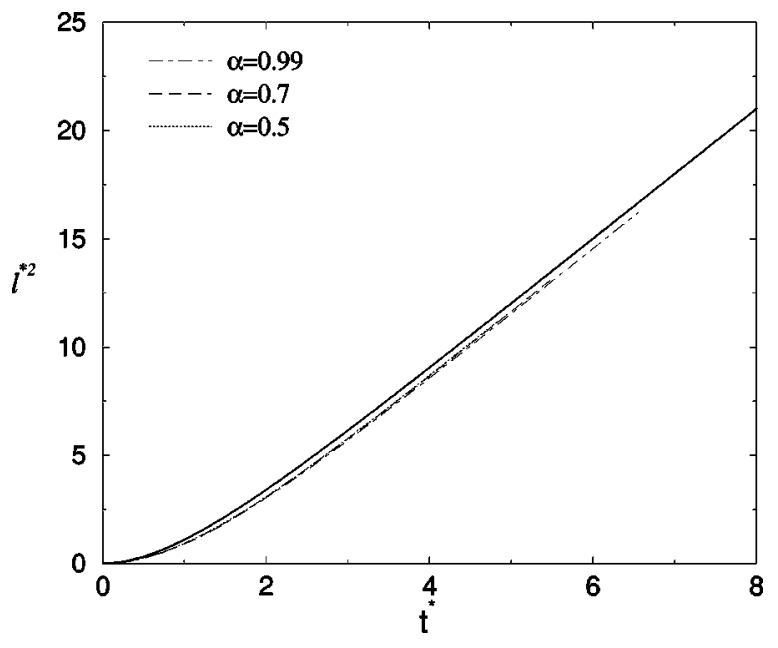

FIG. 4. Time evolution of the mean-square displacement of the tagged particle for three different values of the coefficient of restitution $\alpha$ for collisions between the tagged particle and the gas particles. Time and length are measured in the reduced dimensionless units defined in the main text. The solid line is the theoretical prediction given by Eq. (2.26).

\section{DISCUSSION}

The Fokker-Planck equation, obtained from the Boltzmann-Lorentz equation in the limit of a small ratio of gas to tagged particle mass $\Delta$, allows an exact analysis of the tagged particle dynamics. The surrounding gas is assumed to be in the homogeneous cooling state. In particular, the study shows the clear separation of time scales required for a hydrodynamic description. For tagged particle dynamics the latter refers to diffusion. The objective here has been to confirm the validity of the asymptotic analysis leading to the Fokker-Planck limit by direct Monte Carlo simulation of the Boltzmann-Lorentz equation. The excellent agreement found for the various properties studied provides convincing evidence for the validity of the Fokker-Planck equation and its exact consequences.

The separation of time scales between microscopic (kinetic) and diffusive modes is quite clear, once the complications due to cooling have been suppressed by the change of

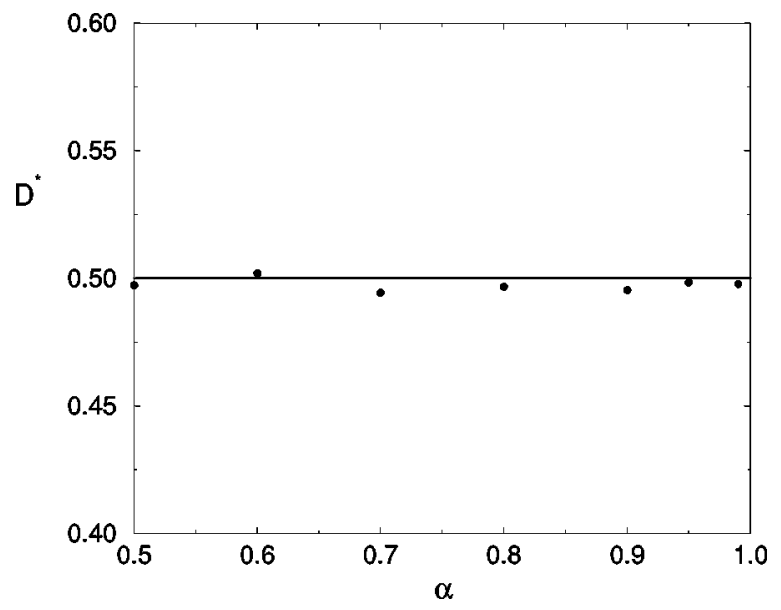

FIG. 5. Comparison between the numerical value of the reduced self-diffusion coefficient obtained from the Boltzmann-Lorentz equation (dots) and the predicted value $D^{*}=1 / 2$ (solid line).

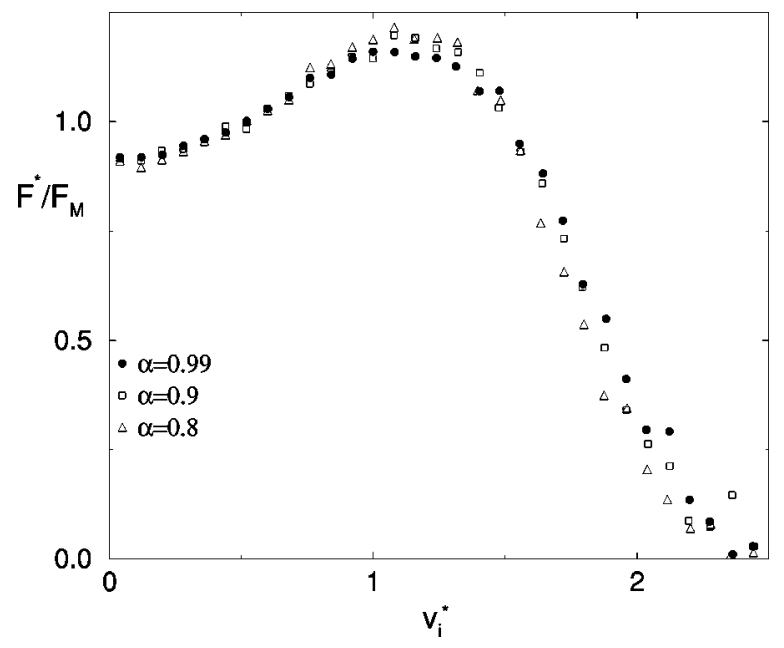

FIG. 6. Distribution function of the tagged particle normalized by the Gaussian for three different values of the restitution coefficient $\alpha$. For the three of them it is $\epsilon>1$, and the Fokker-Planck equation is not expected to hold. The velocity is measured in the reduced units defined in Eq. (2.10).

variables in Eq. (2.10). In the new variables, the description is independent of $\alpha$ and, therefore, applies for arbitrary degree of dissipation for the tagged particle. However, the gas is implicitly restricted to weak dissipation due to the condition $\epsilon<1$, where $\epsilon$ is defined by Eq. (2.11). The explicit form for $\epsilon$ given in the Appendix shows that this condition requires $1-\alpha_{g}^{2} \propto \Delta$. The origin of this condition is a requirement that the ratio of tagged particle temperature to gas temperature does not grow in time, since it appears as a factor of $\Delta$ in the asymptotic analysis [7]. The relevance of this condition for the Fokker-Planck limit is established in Fig. 6 showing the distribution function $F$ normalized by the Gaussian $F_{M}$ for stronger gas dissipation, $\alpha_{g}=0.95$, and for several values of the restitution coefficient for collisions between the tagged particle and the fluid, $\alpha=0.99,0.9$, and 0.8 . The corresponding values for $\epsilon$ are $1.73,1.81$, and 1.915, respectively. The deviations from Maxwellian are now large and the Fokker-Planck description, which predicts a Maxwellian velocity distribution, is no longer valid. Let us stress that the restriction $\epsilon<1$ affects only the Fokker-Planck limit and not the more general picture of approach to diffusion, as described by the Boltzmann-Lorentz equation. This has been demonstrated in Ref. [6], where the mean-square displacement has been simulated for mechanically identical particles for $0.6 \leqslant \alpha_{g} \leqslant 1$, which includes conditions of strong gas particle dissipation and consequently $\epsilon<1$.

All our previous analysis is based on the accuracy of the Boltzmann-Lorentz equation to describe the time evolution of a tagged particle in a low-density gas, when all the collisions are inelastic. For the particular case of self-diffusion the validity of the kinetic equation has been explicitly shown in Ref. [6], by comparing its predictions with the results obtained from molecular-dynamics simulation of a system of inelastic disks. Here we present some additional evidence when the Brownian limit of a massive particle is considered. From a computational point of view, the study of time evolution of a massive particle in a bath, whose state must not be perturbed by the motion of the particle, is a very demanding simulation, requiring a lot of computing time in order to get 


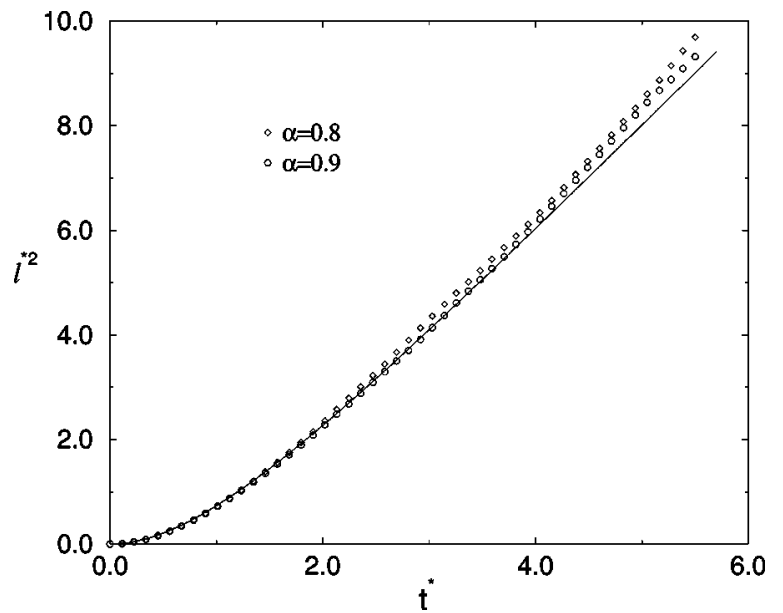

FIG. 7. Time evolution of the mean-square displacement of a tagged disk in a low-density gas of inelastic particles, using molecular dynamics. The mass ratio is $\Delta=2 \times 10^{-2}$ and two values for the coefficient of restitution for collisions of the tagged particles have been considered: $\alpha=0.9$ (squares) and $\alpha=0.8$ (circles). The coefficient of restitution for collisions among fluid particles is $\alpha_{g}$ $=0.99$. Quantities are measured in the dimensionless reduced units defined in the main text. The solid line is the theoretical prediction from the Fokker-Planck description, Eq. (2.26).

a significant level of statistics.

We have simulated a system of hard disks in a square domain with periodic boundary conditions. The gas was composed of 3025 particles and the results we present are averages over 500 trajectories of the Brownian particle, each of them generated by a different computer run. Again the tagged and gas particles were of the same size. The initial condition was generated by running the gas, without tagged particle, for a period of time large enough to allow the system to reach the homogeneous cooling state. Then, one of the gas particles, randomly selected, was substituted by the tagged particle, i.e., its mass was changed to the new value. The simulation technique was based on the "event driven" algorithm [13].

Figure 7 shows the results obtained for the time evolution of the mean-square displacement of the tagged particle in a system with $\alpha_{g}=0.99$ and a mass ratio $\Delta=0.02$. The density of the gas is $n \sigma^{2}=1.322 \times 10^{-3}$, which corresponds to a solid fraction $1.039 \times 10^{-3}$. Two values of the restitution coefficient for collisions of the tagged particle have been considered, $\alpha=0.9$ and $\alpha=0.8$. The solid line is the prediction given by Eq. (2.26). Again a good agreement is obtained, providing a very strong test on the validity of the kinetic equation even before the system reaches the hydrodynamic regime. Similar results were obtained for other values of the parameters of the system, namely those considered in the DSMC results reported in the preceding section. In the figure it is observed that the discrepancy between theory and simulation increases with time. This is a statistics effect which decreases as the number of trajectories increases. This is more clearly seen in Fig. 8, where we have represented the time evolution of the temperature of the tagged particle for the simulations corresponding to $\alpha=0.9$ in Fig. 7. Although the results are consistent with the predictions from the Fokker-Planck equation, the fluctuations are too large to make a detailed quantitative comparison. This is not the case

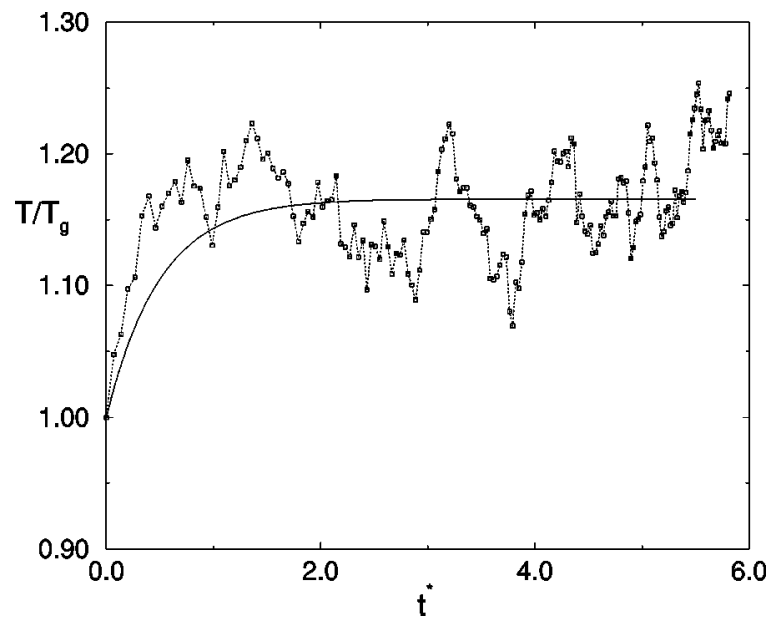

FIG. 8. Time evolution of the temperature of the Brownian particle for the same system as considered in Fig. 7 with $\alpha=0.9$. The temperature is scaled with the time-dependent temperature of the gas. The solid line is the theoretical prediction given by Eq. (2.23). Time is measured in the reduce unit defined in the text.

for the mean-square displacement, as seen in Fig. 7. In this sense, when comparing the results obtained by using the DSMC method with those from MD, we must take into account that the number of trajectories we have considered in the former is 200 times larger than in the latter. Increasing the number of MD trajectories by such a factor would imply a time too long for practical computer simulations.

In summary, a formal limit of the Boltzmann-Lorentz equation for a tagged particle with mass large compared to particles of the surrounding gas leads to a Fokker-Planck kinetic equation. This equation can be mapped onto the corresponding equation for elastic collisions by a change of space and time scales whose exact solution is known. In particular, this equation demonstrates the separation of time scales associated with the rapid transition from complex initial transients to hydrodynamics (diffusion). The property occurs independent of the degree of dissipation in the collisions between the heavy particle and surrounding gas particles. In the present paper, the formal analysis leading to the FokkerPlanck equation has been verified by direct simulation of the Boltzmann-Lorentz equation. Finally, the validity of the Boltzmann-Lorentz equation itself has been confirmed by selected molecular-dynamics simulations.

\section{ACKNOWLEDGMENTS}

The research of J.J.B., M.J.R-M., and R.G-R. was partially supported by the Dirección General de Investigación Científica y Técnica (Spain) through the grant PB98-1124. The research of J.W.D was supported in part by NSF Grant No. PHY 9722133.

\section{APPENDIX: DETAILED FORM FOR $\epsilon$}

The Fokker-Planck limit leading to Eq. (2.8) is restricted to small mass ratio $\Delta$ and $\epsilon<1$. The parameter $\epsilon$ is defined by Eq. (2.11), with the cooling rate $\zeta$ and friction coefficient $\gamma_{e}$ given by Eqs. (2.6) and (2.9), respectively, 


$$
\begin{aligned}
\boldsymbol{\epsilon} & \equiv \frac{\zeta(t)}{2 a \gamma_{e}(t)} \\
& =\frac{\left(1-\alpha_{g}^{2}\right) \Gamma(d / 2)}{16 \Gamma\left(\frac{d+3}{2}\right) \Delta}\left(\frac{\sigma_{g}}{\sigma_{0}}\right)^{d-1} \int d \mathbf{v} \int d \mathbf{v}_{1} g^{3} \phi(\mathrm{v}) \phi\left(\mathbf{v}_{1}\right) \\
& \simeq \frac{1-\alpha_{g}^{2}}{4 \sqrt{2} a \Delta}\left(\frac{\sigma_{g}}{\sigma_{0}}\right)^{d-1}\left[1+\frac{3}{32} c^{*}\left(\alpha_{g}\right)\right] .
\end{aligned}
$$

In the third line the integral has been performed using an approximate solution to the Boltzmann equation obtained by expanding $\phi(\mathrm{v})$ in Sonine polynomials and retaining the first correction to the Gaussian [1,9]. This approximation has been tested via Monte Carlo simulation and is accurate for the relevant velocities within a few percent [12]. The contribution $c^{*}\left(\alpha_{g}\right)$ above is due to this first correction and is given by

$$
c^{*}\left(\alpha_{g}\right)=\frac{32\left(1-\alpha_{g}\right)\left(1-2 \alpha_{g}^{2}\right)}{9+24 d+(8 d-41) \alpha_{g}+30 \alpha_{g}^{2}\left(1-\alpha_{g}\right)} .
$$

The condition $\epsilon<1$ restricts the range of $\alpha_{g}$ to that for weak dissipation in the gas since $\Delta$ must be small. The de- tailed values can be adjusted by considering particles of very different sizes. For simplicity, the sizes have been taken equal in the simulations reported in Sec. III. Then, for the chosen values in the DSMC method in Sec. III, $\alpha_{g}=0.99$ and $\Delta=10^{-2}$, the above expression for $\epsilon$ simplifies to

$$
\epsilon \simeq \frac{0.704}{1+\alpha},
$$

where we have also particularized for $d=3$. Clearly $\epsilon<1$ for all $0 \leqslant \alpha \leqslant 1$ and the Fokker-Planck equation is expected to hold for arbitrary inelasticity of the collisions of the tagged particle with the gas particles.

In the reported MD simulations, we used hard disks ( $d$ =2), $\Delta=2 \times 10^{-2}$, and $\alpha_{g}=0.99$. This leads to

$$
\epsilon \simeq \frac{0.351}{1+\alpha}
$$

and, again, the condition $\epsilon<1$ is fulfilled for arbitrary values of $\alpha$.

When carrying out the simulations, we have observed that the agreement with the theoretical predictions inproves as the value of $\epsilon$ decreases, although the validity of the theory requires just $\epsilon<1$.
[1] A. Goldshtein and M. Shapiro, J. Fluid Mech. 282, 75 (1995).

[2] N. Sela and I. Goldhirsch, J. Fluid Mech. 361, 41 (1998).

[3] J.J. Brey, J.W. Dufty, C-S. Kim, and A. Santos, Phys. Rev. E 58, 4638 (1998).

[4] V. Garzo and J.W. Dufty, Phys. Rev. E 59, 5895 (1999).

[5] M-L. Tan and I. Goldhirsch, Phys. Rev. Lett. 81, 3022 (1998); L.P. Kadanoff, Rev. Mod. Phys. 71, 435 (1999).

[6] J.J. Brey, M.J. Ruiz-Montero, D. Cubero, and R. Garcia-Rojo, Phys. Fluids (to be published).

[7] J.J. Brey, J.W. Dufty, and A. Santos, J. Stat. Phys. 97, 281 (1999).
[8] J.J. Brey, J.W. Dufty, and A. Santos, J. Stat. Phys. 87, 1051 (1997).

[9] T.P.C. van Noije and M.H. Ernst, Granular Matter 1, 57 (1998).

[10] J. A. McLennan, Introduction to Nonequilibrium Statistical Mechanics (Prentice-Hall, Englewood Cliffs, NJ, 1989).

[11] G. Bird, Molecular Gas Dynamics and the Direct Simulation of Gas Flows (Clarendon Press, Oxford, 1994).

[12] J.J. Brey, M.J. Ruiz-Montero, and D. Cubero, Phys. Rev. E 54, 3664 (1996).

[13] M.P. Allen and D.J. Tildesley, Computer Simulation of Liquids (Oxford University Press, Oxford, 1987). 